\title{
Graphitization and Growth of free-standing Nanocrystalline Graphene using In Situ Transmission Electron Microscopy
}

C.N. Shyam Kumar ${ }^{1,2}$, Venkata Sai Kiran Chakravadhanula,3, Adnan Riaz ${ }^{1,2}$, Simone Dehm ${ }^{1}$, Di Wang ${ }^{1}$, Xiaoke Mu ${ }^{1}$, Ralph Krupke ${ }^{1,2}$, Christian Kübel ${ }^{1,3,4}$

${ }^{1}$ Institute of Nanotechnology, Karlsruhe Institute of Technology, 76021 Karlsruhe, Germany

${ }^{2}$ Department of Materials and Earth Sciences, Technische Universität Darmstadt, 64287 Darmstadt, Germany

${ }^{3}$ Helmholtz Institute Ulm, 89081 Ulm, Germany

${ }^{4}$ Karlsruhe Nano Micro Facility, Karlsruhe Institute of Technology, 76021 Karlsruhe, Germany

Graphitization of polymers is an effective way to synthesize nanocrystalline graphene on different substrates with tunable shape, thickness and properties [1]. The catalyst free synthesis results in crystallite sizes on the order of few nanometer, significantly smaller compared to commonly prepared polycrystalline graphene [2]. Even though this method provides the flexibility of graphitizing polymer films on different substrates, substrate free graphitization of freestanding polymer layers has not been studied yet. For the first time, we report on the thermally induced graphitization and domain growth of free standing nanocrystalline graphene thin films using in situ TEM techniques to study the different processes contributing to the graphene growth.

The photo resist used as precursor for the nanocrystalline graphene preparation is the commercially available microposit S1085. The resist was spin coated onto a MEMs based heating chip (Protochips Inc.) (figure 1a). The initial heating was carried out ex situ at $10^{-7} \mathrm{mbar}$ at a temperature of $600{ }^{\circ} \mathrm{C}$ for 5 hours. The resulting thin films were analyzed by local Raman spectroscopy, confirming a uniform carbonization over the heating area (figure 1b). Free standing partially graphitized films had formed across the holes of the supporting film on the heating chip, confirming that a substrate free graphitization is possible. The Raman signature of the free standing film (peak positions and $\mathrm{I}_{\mathrm{D}} / \mathrm{I}_{\mathrm{G}}$ ratio) is almost identical to a film graphitized on a $\mathrm{SiO}_{2}$ terminated $\mathrm{Si}$ wafer (figure 1c). This confirms that graphitization without any catalytic support of the substrate yields very similar materials. These free standing films were the starting point for a detailed in situ TEM graphitization study at temperatures up to $1200^{\circ} \mathrm{C}$.

In situ TEM observations of the free standing carbonized films were carried out using high resolution transmission electron microscopy (HRTEM), selected area electron diffraction (SAED) and electron energy loss spectroscopy (EELS) techniques to analyze the graphitization with temperature by looking at domain structure \& size, crystallinity, and $\mathrm{sp}^{2} / \mathrm{sp}^{3}$ ratio. The analysis showed that the growth of the nanocrystalline domains in the intermediate temperature range of $600^{\circ} \mathrm{C}-1000^{\circ} \mathrm{C}$ mainly proceeded by consuming amorphous carbon present around the domains. After heating to $1000^{\circ} \mathrm{C}$, essentially only $\mathrm{sp}^{2}$ carbon (figure $2 \mathrm{~g}$ ) is left in the [001] texture dominated film (figure 2e). Further growth of the domains at higher temperatures $\left(1000-1200{ }^{\circ} \mathrm{C}\right)$ was facilitated by the merging of small crystalline domains forming bigger ones. The merging of domains occurred both by migration of smaller domains on the surface of larger ones as well as around the edges to the vacuum leading to an extension of the larger domain underneath. However, both mobile and stationary (pinned) domains with varying size and shape were observed. The partially stacked graphene layers exhibit a preferential misorientation of $22^{\circ}$ (figure 2i). Further details of the processes observed in situ will be discussed in the presentation. 


\section{References:}

[1] Z. Zhang, B. Ge, Y. Guo, D. Tang, X. Wang and F. Wang, Chem. Commun., 2013, 49, 2789.

[2] A. Riaz, F. Pyatkov, A. Alam, S. Dehm, A. Felten, V. S. K. Chakravadhanula, B. S. Flavel, C. Kübel, U. Lemmer and R. Krupke, Nanotechnology, 2015, 26, 325202.

[3] C.N. Shyam Kumar greatly acknowledge $\mathrm{PhD}$ funding from Deutscher Akademischer Austauschdienst (DAAD)

(a)

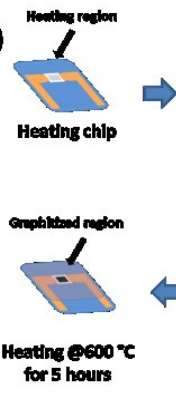

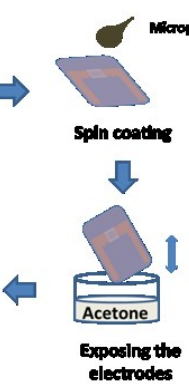
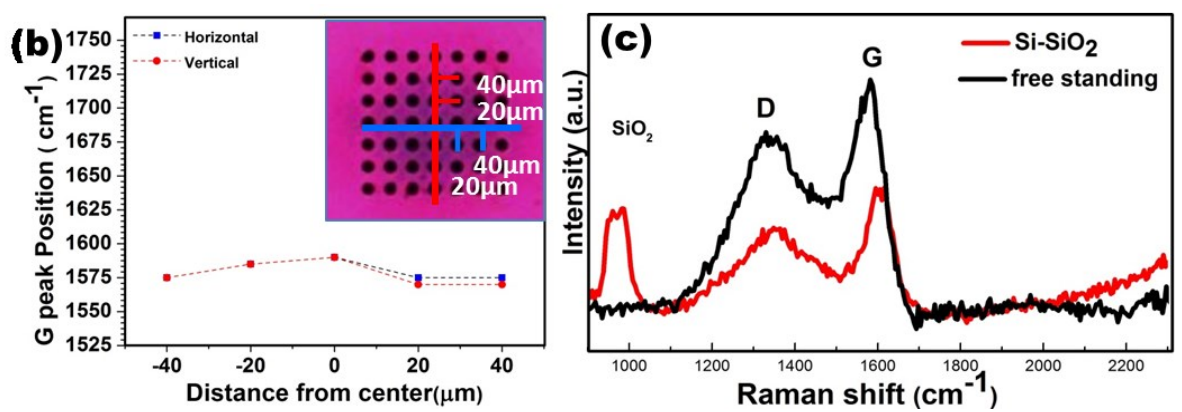

Figure 1. (a) Schematic of the sample preparation procedure, (b) $\mathrm{G}$ peak position in the active area of the heating chip, (c) Raman spectra of free standing and $\mathrm{SiO}_{2}$ supported film at $600{ }^{\circ} \mathrm{C}$.
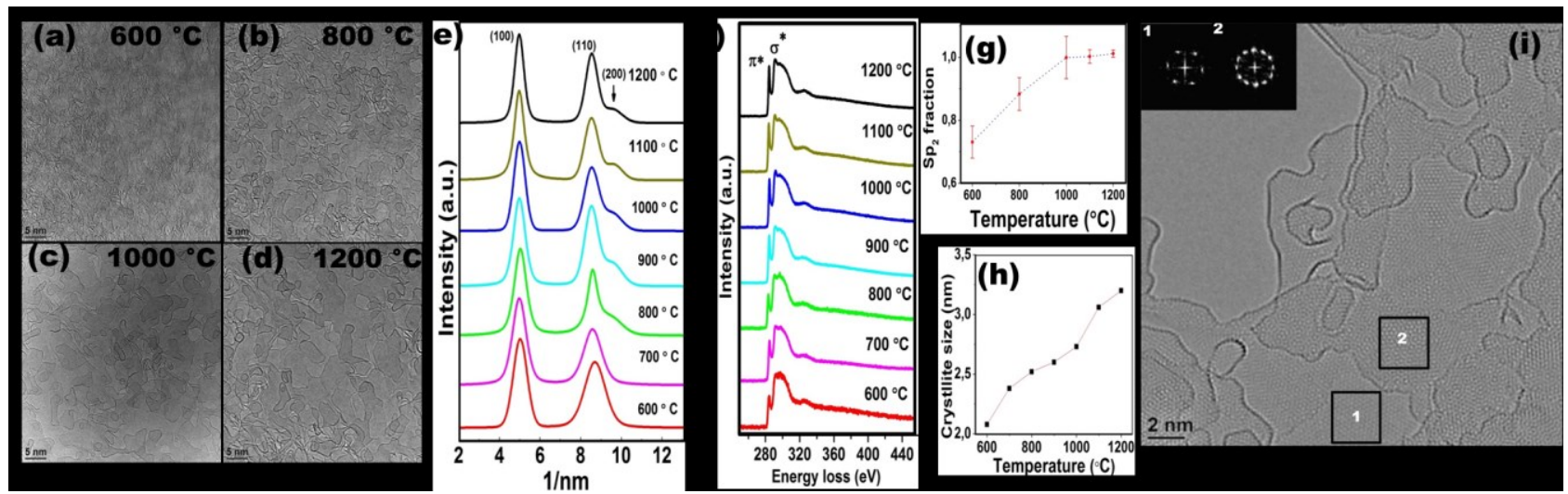

Figure 2. (a-d) Evolution of nanocrystalline domains with temperature, (e) and (f) SAED intensity profiles and carbon core loss EELS edge at different temperatures, (g) crystallite size evolution and (h) $\mathrm{sp}^{2}$ fraction at different temperatures, (i) HRTEM image of the graphitized layers, FFT from the marked regions showing a misorientation of $22^{\circ}$ is given in inset. 